\title{
A Unifying Approximate Potential for Weighted Congestion Games
}

\author{
Yiannis Giannakopoulos ${ }^{\dagger} \quad$ Diogo Poças ${ }^{\ddagger}$
}

March 15, 2022

\begin{abstract}
We provide a unifying, black-box tool for establishing existence of approximate equilibria in weighted congestion games and, at the same time, bounding their Price of Stability. Our framework can handle resources with general costs-including, in particular, decreasing ones-and is formulated in terms of a set of parameters which are determined via elementary analytic properties of the cost functions.

We demonstrate the power of our tool by applying it to recover the recent result of Caragiannis and Fanelli [ICALP'19] for polynomial congestion games; improve upon the bounds for fair cost sharing games by Chen and Roughgarden [Theory Comput. Syst., 2009]; and derive new bounds for nondecreasing concave costs. An interesting feature of our framework is that it can be readily applied to mixtures of different families of cost functions; for example, we provide bounds for games whose resources are conical combinations of polynomial and concave costs.

In the core of our analysis lies the use of a unifying approximate potential function which is simple and general enough to be applicable to arbitrary congestion games, but at the same time powerful enough to produce state-of-the-art bounds across a range of different cost functions.
\end{abstract}

\section{Introduction}

Atomic congestion games are one of the most well-studied topics in algorithmic game theory [Rou16, NRTV07]. In their most general form, players have weights and compete over a common set of resources; the cost of each resource is a function of the total weight of the players that end up using it. As a result, they can model a wide range of interesting applications including, e.g., network routing [Rou07] and load balancing [Vöc07], but also even cost-sharing games (via the use of decreasing cost functions) like fair network design [TW07].

An important special case is that of unweighted congestion games, where the costs depend only on the number of players that use each edge. In a seminal paper, Rosenthal [Ros73] proved that unweighted congestion games always have (pure Nash) equilibria. A key tool in his derivation was the novel use of a potential function, which is able to capture the different players' deviations in a very elegant and concise way. Then, the desired equilibrium is derived as the minimizer of that function (over all feasible outcomes of the game). This technique can also be viewed as an equilibrium refinement, and has been a very influential idea in game theory [MS96]. It allows us not only to establish the

\footnotetext{
${ }^{*}$ Part of this work was done while the authors were members of the Operations Research group at Technical University of Munich, School of Management, supported by the Alexander von Humboldt Foundation with funds from the German Federal Ministry of Education and Research (BMBF). D. Poças was also supported by FCT via LASIGE Research Unit, ref. UIDB/00408/2020 and ref. UIDP/00408/2020.

A preliminary version of this paper appeared in SAGT'20 [GP20].

${ }^{\dagger}$ Friedrich-Alexander-Universität Erlangen-Nürnberg, Department of Data Science (yiannis.giannakopoulos@fau.de)

¥LASIGE, Faculdade de Ciências, Universidade de Lisboa (dmpocas@fc.ul.pt)
} 
existence of equilibria, but in many cases, this special potential-minimizer equilibrium has additional desired properties.

Of particular importance to us in this paper, is that it has been the de facto method for proving Price of Stability (PoS) bounds in congestion games (see, e.g., [NRTV07, Ch. 18, 19]). The PoS notion [ADK ${ }^{+} 08$, CSSM04] captures the minimum approximation ratio of the social cost, among all equilibria, to the socially optimum outcome of the game (that might not be an equilibrium). In other words, the PoS is the best-case counterpart of the notorious Price of Anarchy (PoA) notion introduced by Koutsoupias and Papadimitriou [KP99, Pap01]

Unfortunately, though, it is a well-known fact that general weighted congestion games do not always have equilibria and thus, do not admit a potential function. To alleviate this, a line of work has focused on designing approximate potential functions (see, e.g., [CR09, HKS14, CKS11, CGGS19, CF19]): the minimizer of such functions is guaranteed to be an approximate equilibrium (as opposed to an exact one that is given by Rosenthal's potential in the unweighted case), while at the same time it can achieve a good approximation ratio to the optimal social cost (providing, thus, an upper bound for the approximate-equilibrium extension of the PoS notion). However, most of those prior works use different approximate potentials, designed specially for the particular cost-function model that each one studies.

Our goal in this paper is to provide a simple, high-level framework whose interface is agnostic to the underlying potential function technicalities and which can readily be instantiated for all resource costs at hand to derive meaningful bounds.

\subsection{Related Work}

Following the seminal work of [Ros73], a long line of results has been devoted to the (non)existence of equilibria in weighted congestion games. [GMV05, LO01, FKS05] demonstrated that equilibria might not exist even in very simple classes of games, including network congestion games with quadratic cost functions and games where player weights are either 1 or 2. On the other hand, [FKS05, PS07, HK12] showed that equilibria do exist in games with affine or exponential cost functions; [FKK ${ }^{+} 09, \mathrm{HKM} 12$ ] proved the same for singleton games (where players can only occupy single resources). Dunkel and Schulz [DS08] were able to extend the nonexistence instance of Fotakis et al. [FKS05] to a hardness gadget, in order to show that, deciding whether a congestion game with step cost functions has an equilibrium, is a (strongly) NP-complete problem.

Regarding the existence of approximate equilibria in general weighted congestion games, [CGG 20 ] showed that games with $n$ players always have $n$-approximate equilibria, and this guarantee is tight (up to logarithmic factors); they also proved that the corresponding decision problem, i.e., of the existence of $\tilde{\Theta}(n)$-approximate equilibria, is NP-complete.

A lot of work has been focused on the important special case of polynomial congestions games, parameterized by the maximum degree $d$ of the cost functions. Although, due to [FKS05] we already know that exact equilibria do not in general exist in such games, Caragiannis et al. [CFGS11] were the first to show that $\alpha$-approximate equilibria do exist for $\alpha=d$ !; this factor was later improved to $\alpha=d+1$ [HKS14, CGGS19] and $\alpha=d$ [CF19]. As a matter of fact, Caragiannis and Fanelli [CF19] provide an even more comprehensive result that, for any choice of a parameter $\delta \in[0,1]$, simultaneously establishes the existence of $(d+\delta)$-approximate equilibria and gives an upper bound of $\frac{d+1}{\delta+1}$ on their PoS. They achieve this by designing an appropriate approximate potential function, tailored to polynomial costs. On the nonexistence front, [HKS14] first gave instances of very simple, two-player polynomial congestion games that do not have $\alpha$-approximate equilibria, for $\alpha \approx 1.153$. This was recently improved to $\alpha=\Omega\left(\frac{\sqrt{d}}{\log d}\right)$ by Christodoulou et al. [CGG ${ }^{+} 20$ ], who also established NP-hardness of the corresponding existence decision problem.

The work of Hansknecht et al. [HKS14] is very relevant for our approach in this paper, since they also propose a "generic" approximate potential function that can, in principle, be applied to general cost functions. They instantiate it for polynomial costs to derive their aforementioned existence of $(d+1)$ - 
approximate equilibria. Additionally, they also state a result about the existence of $\frac{3}{2}$-approximate equilibria in games with nondecreasing concave costs; however, this proof in their paper is not complete. Furthermore, [HKS14] focuses just on the existence of approximate equilibria, and thus it does not provide any PoS bounds.

Another well-studied class of congestion games is that of fair cost sharing, where each resource has a constant initial cost which is split equally among the players that use it. Thus, such games have decreasing cost functions. Finding the PoS for the special, undirected network version of such games is a notorious open problem in the field (see, e.g., [ $\left.\left.\mathrm{ADK}^{+} 08, \mathrm{FKL}^{+} 06, \mathrm{BCFM} 13, \mathrm{BFM} 14, \mathrm{FHP} 16\right]\right)$. Very relevant for us is the work of Chen and Roughgarden [CR09] who showed that general weighted fair cost sharing games always have $\alpha$-approximate equilibria whose PoS is at most $O\left(\frac{\log W}{\alpha}\right)$, for any choice of parameter $\alpha=\Omega\left(\log w_{\max }\right)$, where $w_{\max }$ is the maximum weight of any player and $W$ the maximum possible load in any resource. They achieve this by designing a special approximate potential function, tailored to the specific form of the cost functions.

\subsection{Our Results and Techniques}

We propose a new approximate potential function (see (9)) for weighted congestion games with general cost functions. In particular, our potential can be instantiated beyond the standard model of polynomial cost functions and the common assumption of non-decreasing monotonicity. However, this potential is only used in the analysis part of our paper: we hide away its specific form by hard-coding it within the proof of a unifying tool (Theorem 1). Then, this tool can be used in a black-box way to readily derive both existence of approximate equilibria, and bounds on their PoS. This proof makes also use of a general, high-level lemma that can capture the essence of the potential method as a technique for deriving existence and PoS bounds for approximate equilibria (Lemma 1); we believe this might be of independent interest, since in future work it could be used for alternative potential functions, beyond our choice of (9) in this paper.

Our framework effectively works in two steps. Given a congestion game, first one has to determine how good its cost functions are with respect to two simple, analytic properties (Definition 2). Then, the resulting "goodness" parameters can be plugged straight into our master theorem (Theorem 1) to deduce the existence of an $(\alpha, \beta)$-equilibrium; that is, an $\alpha$-approximate (pure Nash) equilibrium whose social cost is at most a factor of $\beta$ away from the optimum.

We demonstrate the power of our tool by applying it to recover and improve prior bounds on the existence of $(\alpha, \beta)$-equilibria for well-studied classes of congestion games, as well as to derive novel results. The simplicity and the algebraic nature of our tool allows us to produce fine-grained bounds in the form of a parametric trade-off curve that describes the relation between the $\alpha$ and $\beta$ parameters of the $(\alpha, \beta)$-equilibrium; in other words, all our results give a continuum of existence bounds. Our bounds are summarized in Table 1.

More specifically, first (Theorem 2) we rederive the recent bounds of [CF19] for polynomial congestion games, in a more "clean", high-level way. Then (Theorem 4), we improve the $\alpha, \beta$ parameters on the $(\alpha, \beta)$-equilibrium existence results of [CR09] for fair cost-sharing games (a more detailed comparison can be seen in Fig. 1). Furthermore, we derive new results for (nondecreasing) concave costs: we show that $\left(\lambda, \frac{\lambda}{\lambda-1}\right)$-equilibria always exist, for all $\lambda \in\left[\frac{3}{2}, 2\right]$ (Theorem 3). The special corner case of a $\left(\frac{3}{2}, 3\right)$-equilibrium is compatible, thus, with the $\frac{3}{2}$-approximate equilibrium existence stated in [HKS14].

Another interesting characteristic of our tool is its modularity: it can readily combine different cost functions to give bounds for more complex congestion games (see Definition 3). For example, we prove that games with cost functions that are conical combinations of $d$-degree polynomials and concave costs, always have $\left(\lambda, 1+\frac{d+1}{\lambda}\right)$-equilibria, where $\lambda$ ranges in $[d, d+1]$ (Theorem 5).

Finally, an added advantage of our black-box method is that it also results in arguably simpler and more streamlined proofs for the existence and PoS bounds.

Before concluding the overview of our results, we want to elaborate a bit more on the comparison to the potential approach of Hansknecht et al. [HKS14]. Although [HKS14] does not deal with PoS bounds, 


\begin{tabular}{|c|c|c|c|}
\hline \multirow[b]{2}{*}{ Cost functions } & \multirow[b]{2}{*}{ Previous Work } & \multicolumn{2}{|c|}{ Our Results } \\
\hline & & General & Extreme Points \\
\hline Polynomials of degree $\leq d$ & $\left(\lambda, \frac{d+1}{\lambda}\right)$, for $\lambda \in[d, d+1]$ & $\left(\lambda, \frac{d+1}{\lambda}\right), \quad$ for $\lambda \in[d, d+1]$ & $\left(d, 1+\frac{1}{d}\right),(d+1,1)$ \\
\hline Concave & $\begin{array}{r}{[\mathrm{CF} 19]} \\
\left(\frac{3}{2}, \infty\right) \quad[\mathrm{HKS} 14]\end{array}$ & $\begin{array}{r}\text { [Theorem 2] } \\
\left(\lambda, \frac{\lambda}{\lambda-1}\right), \quad \begin{array}{r}\text { for } \lambda \in\left[\frac{3}{2}, 2\right] \\
\text { [Theorem 3] }\end{array}\end{array}$ & $\left(\frac{3}{2}, 3\right),(2,2)$ \\
\hline Polynomials + Concave & $\mathrm{N} / \mathrm{A}$ & $\left(\lambda, 1+\frac{d+1}{\lambda}\right)$, for $\lambda \in[d, d+1]$ & $\left(d, 2+\frac{1}{d}\right),(d+1,2)$ \\
\hline Fair cost sharing & $\begin{array}{l}\left(\lambda, 1+\frac{2 \log _{2}(1+W)}{\lambda}\right) \\
\text { for } \lambda=\Omega\left(\ln w_{\max }\right) \\
{[\text { CR09] }}\end{array}$ & $\begin{array}{l}\quad \text { [Theorem 5] } \\
\left(\Theta\left(\ln w_{\max }\right)+\lambda, 1+\frac{\ln W}{\lambda}\right) \\
\text { for } \lambda \geq 1 \quad \text { [Theorem 4] }\end{array}$ & $\begin{array}{l}\left(\Theta\left(\ln w_{\max }\right), 1+\ln W\right), \\
(\Theta(\ln W), \Theta(1))\end{array}$ \\
\hline
\end{tabular}

Table 1: Our main results on the existence of $(\alpha, \beta)$-equilibria for different cost models. For polynomials of degree $d$ we recover the result of [CF19]. For fair costs our results improve those of [CR09] and for concave costs we extend those of [HKS14]. For mixtures of different cost functions, namely polynomial and concave, our results are novel.

as far as existence of approximate equilibria is concerned, their paper is rather similar in principle to ours. They propose a general potential function which is based on a discrete interpretation of the cost function's integral, which corresponds to the first component of our potential in (9). We take a different approach by using directly the actual integral, and also adding an extra term that corresponds to a weighted average of the costs of the players' weights. In that way, we avoid a lot of the intricate technicalities that are involved with the discrete arguments (e.g., orderings of the weights) in [HKS14], making the application of our potential (via our high-level tool of Theorem 1) more "tractable" for a wider range of cost functions.

\section{Model and Notation}

We use $\mathbb{R}_{+}$to denote the set of nonnegative real numbers.

In a (weighted) congestion game $\mathcal{G}$ there are finite, nonempty sets of players $N$ and resources $E$. Let $n=|N|$. Each player $i \in N$ has a weight $w_{i} \in \mathbb{R}_{+}$and a strategy set $S_{i} \subseteq 2^{E}$. We use $w_{\min }=\min _{i \in N} w_{i}$ and $w_{\max }=\max _{i \in N} w_{i}$ for the minimum and maximum player weights, respectively, and for a subset of players $I \subseteq N$, we use $w_{I}=\sum_{i \in I} w_{i}$ to denote the sum of their weights. For the special case of $w_{\min }=w_{\max }=1$, that is, if all weights are 1 , we say that $\mathcal{G}$ is unweighted.

Associated with each resource $e \in E$ is a cost function $c_{e}: \mathbb{R}_{+} \longrightarrow \mathbb{R}_{+}$. In general, we will make no extra assumptions on the cost functions. However, important special cases, that we will also study as applications of the main tool of our paper, include polynomial congestion games of degree $d$, for $d \geq 1$ integer, and fair cost sharing games. In the former, the cost functions are polynomials with nonnegative coefficients and degree at most $d$; in the latter, cost functions are (decreasing) of the form $c_{e}(x)=\frac{a_{e}}{x}$ where $a_{e}$ is a positive real.

A (pure) strategy profile (or outcome) is a choice of strategies $s=\left(s_{1}, s_{2}, \ldots, s_{n}\right) \in \boldsymbol{S}=S_{1} \times \cdots \times S_{n}$. We use the standard game-theoretic notation $\boldsymbol{s}_{-i}=\left(s_{1}, \ldots, s_{i-1}, s_{i+1}, \ldots s_{n}\right), \boldsymbol{S}_{-i}=S_{1} \times \cdots \times S_{i-1} \times S_{i+1} \times$ $\cdots \times S_{n}$. In that way, for example, we can denote $s=\left(s_{i}, s_{-i}\right)$. Given a profile $s \in S$, we define the load $x_{e}(s)$ of resource $e$ as the total weight of players that use resource $e$ at outcome $s$, i.e., $x_{e}(s)=$ $w_{N_{e}(s)}=\sum_{i \in N: e \in s_{i}} w_{i}$, where $N_{e}(s)$ is the set of players using $e$. We will use $W=\sum_{i \in N} w_{i}$ to denote the maximum possible load of any resource. The cost of player $i$ is defined by $C_{i}(s)=\sum_{e \in s_{i}} c_{e}\left(x_{e}(s)\right)$. The social cost of a strategy profile $s$ is the weighted sum of the players' costs

$$
C(s)=\sum_{i \in N} w_{i} \cdot C_{i}(s)=\sum_{e \in E} x_{e}(s) \cdot c_{e}\left(x_{e}(s)\right) .
$$

We use $\mathrm{OPT}(\mathcal{G})=\min _{\boldsymbol{s} \in S} C(s)$ to denote the optimum social cost over all outcomes.

An outcome $s$ is an $\alpha$-approximate (pure Nash) equilibrium, for $\alpha \geq 1$, if

$$
C_{i}(s) \leq \alpha \cdot C_{i}\left(s_{i}^{\prime}, s_{-i}\right) \quad \text { for all } i \in N, s_{i}^{\prime} \in S_{i}
$$


That is, no player can unilaterally deviate from $s$ and improve her cost by more than a factor of $\alpha$. Notice that for the special case of $\alpha=1$ we get the definition of the standard, exact pure Nash equilibrium. We denote the set of all $\alpha$-equilibria of $\mathcal{G}$ by $\mathrm{NE}_{\alpha}(\mathcal{G})$ Then, the $\alpha$-approximate Price of Stability $(\alpha-P o S)$ of $\mathcal{G}$ is the social cost of the best-case Nash equilibrium over the optimum social cost:

$$
\operatorname{PoS}_{\alpha}(\mathcal{G})=\min _{s \in \mathrm{NE}_{\alpha}(\mathcal{G})} \frac{C(s)}{\mathrm{OPT}(\mathcal{G})} .
$$

For $\alpha=1$ we get the standard definition of the Price of Stability (PoS) for exact equilibria [ADK ${ }^{+} 08$ ]. We combine the notions of an approximate equilibrium with approximating the optimum social cost in the following definition:

Definition $1((\alpha, \beta)$-equilibrium). Fix a congestion game $\mathcal{G}$. A strategy profile $s$ is an $(\alpha, \beta)$-equilibrium if it is an $\alpha$-approximate equilibrium of $\mathcal{G}$ (see (1)) and its social cost is at most $\beta$ times the optimal cost of $\mathcal{G}$, i.e., $C(s) \leq \beta \cdot \operatorname{OPT}(\mathcal{G})$.

Notice that if a game has an $(\alpha, \beta)$-equilibrium then, due to (2), its $\alpha$-PoS is at most $\beta$.

\subsection{Equivalent Cost Functions}

It is not difficult to see that, in any weighted congestion game, the cost functions of each resource are actually evaluated on finitely many points: although our model assumes $c_{e}$ to be defined over the entire $\mathbb{R}_{+}$, its values outside the domain $\left\{x_{e}(s) \mid s \in \boldsymbol{S}\right\}$ are irrelevant. In particular, this domain is included within the set of different sums of weights

$$
\mathcal{W}=\left\{\sum_{i \in N} y_{i} \cdot w_{i} \mid y_{i} \in\{0,1\}, i \in N\right\} .
$$

This means that one only needs to define costs on at most $|\mathcal{W}| \leq 2^{n}$ different values: any two games whose costs coincide on $\mathcal{W}$ are equivalent.

However, it is still convenient to treat our costs as functions over $\mathbb{R}_{+}$. First, because this allows for simple and succinct representations. But of particular importance to us, is also the fact that our main tool (Theorem 1) can be applied to all integrable cost functions (so that Definition 2 can be utilized). From the above discussion, it should be obvious that any congestion game has (infinitely) many equivalent representations, that is, different extensions from $\mathcal{W}$ to $\mathbb{R}_{+}$. Such an extension can always be done in a way that $c_{e}$ is an integrable function (since $\mathcal{W}$ is finite).

It is interesting to point out here that different representations can potentially give different existence and PoS bounds via our tool. Although we do not deal with this feature for most of the paper, it is important for our fair cost sharing results (Section 4.3); since function $x \mapsto 1 / x$ is not integrable over the interval $\left[0, w_{\min }\right.$ ) (and as a matter of fact, not even defined on $x=0$ ) we have the freedom, according to the discussion above, to redefine it in any way we want on $\left[0, w_{\min }\right)$, so that it is a well-defined, integrable function over $\mathbb{R}_{+}$.

\section{The Main Tool}

In this section we present our framework for establishing existence of $(\alpha, \beta)$-equilibria in weighted congestion games with general cost functions. We begin with the following lemma, that tries to distil and abstract the potential method technique in congestion games. Specialized or restricted forms of it have essentially been used, even if not explicitly stated, in multiple works in the past (see, e.g., [CR09, HKS14, CF19]). It can be seen as a more fine-grained version of [CGGS19, Lemma 4.1], although some extra care is needed to adapt it to the more abstract setting of our paper and utilize its full power. 
Lemma 1 (Potential Method). Fix a congestion game. Assume that, for each resource e, there exist positive reals $\alpha_{1, e}, \alpha_{2, e}, \beta_{1, e}, \beta_{2, e}$, and a function $\phi_{e}: 2^{N} \longrightarrow \mathbb{R}$ such that $\phi_{e}(\emptyset)=0$ and

$$
\begin{array}{cc}
\alpha_{1, e} \leq \frac{\phi_{e}(I \cup\{i\})-\phi_{e}(I)}{w_{i} \cdot c_{e}\left(w_{I}+w_{i}\right)} \leq \alpha_{2, e} & \text { for all } i \in N, I \subseteq N \backslash\{i\} ; \\
\beta_{1, e} \leq \frac{\phi_{e}(I)}{w_{I} \cdot c_{e}\left(w_{I}\right)} \leq \beta_{2, e} \quad & \text { for all } \emptyset \neq I \subseteq N .
\end{array}
$$

Then the game has an $(\alpha, \beta)$-equilibrium with

$$
\alpha=\max _{e \in E} \frac{\alpha_{2, e}}{\alpha_{1, e}} \quad \text { and } \quad \beta=\frac{\max _{e \in E} \beta_{2, e} / \alpha_{1, e}}{\min _{e \in E} \beta_{1, e} / \alpha_{1, e}} .
$$

Proof. Define function $\Phi(s)=\sum_{e \in E} \frac{1}{\alpha_{1 e}} \phi_{e}\left(N_{e}(s)\right)$ over all feasible outcomes. We will show that $\Phi$ can serve as a desired approximate potential function for our game; that is, for any profiles $s, s^{\prime}$ and any player $i$, it satisfies:

$$
\begin{array}{ccl}
\Phi(s) \leq \Phi\left(s_{i}^{\prime}, s_{-i}\right) & \Longrightarrow \quad C_{i}(s) \leq \alpha \cdot C_{i}\left(s_{i}^{\prime}, s_{-i}\right) \\
\Phi(s) \leq \Phi\left(s^{\prime}\right) \quad \Longrightarrow \quad C(s) \leq \beta \cdot C\left(s^{\prime}\right)
\end{array}
$$

This would be enough to establish our lemma: any (global) minimizer of $\Phi$ is an $\alpha$-approximate equilibrium, due to (5), and at the same time, due to (6), its social cost is within a factor of $\beta$ from the social cost of any other profile (and, thus, from the optimal one). Notice also, that such a minimizer always exists, since the set $\boldsymbol{S}$ of feasible outcomes is finite.

For (5) first, denote for simplicity $N_{e}=N_{e}(s), x_{e}=x_{e}(s)$ and $N_{e}^{\prime}=N_{e}\left(s_{i}^{\prime}, s_{-i}\right), x_{e}^{\prime}=x_{e}\left(s_{i}^{\prime}, s_{-i}\right)$ for all facilities $e$. Then, we have

$$
\begin{aligned}
\Phi\left(s_{i}^{\prime}, s_{-i}\right)-\Phi(s) & =\sum_{e \in E} \frac{1}{\alpha_{1, e}}\left[\phi_{e}\left(N_{e}^{\prime}\right)-\phi_{e}\left(N_{e}\right)\right] \\
& =\sum_{e \in s_{i}^{\prime} \backslash s_{i}} \frac{1}{\alpha_{1, e}}\left[\phi_{e}\left(N_{e} \cup\{i\}\right)-\phi_{e}\left(N_{e}\right)\right]+\sum_{e \in s_{i} \backslash s_{i}^{\prime}} \frac{1}{\alpha_{1, e}}\left[\phi_{e}\left(N_{e} \backslash\{i\}\right)-\phi_{e}\left(N_{e}\right)\right] \\
& \leq \sum_{e \in s_{i}^{\prime} \backslash s_{i}} \frac{\alpha_{2, e}}{\alpha_{1, e}} w_{i} c_{e}\left(x_{e}+w_{i}\right)-\sum_{e \in s_{i} \backslash s_{i}^{\prime}} w_{i} c_{e}\left(x_{e}\right) \\
& \leq w_{i}\left[\alpha \sum_{e \in s_{i}^{\prime} \backslash s_{i}} c_{e}\left(x_{e}+w_{i}\right)-\sum_{e \in s_{i} \backslash s_{i}^{\prime}} c_{e}\left(x_{e}\right)\right] \\
& \leq w_{i}\left[\alpha\left(\sum_{e \in s_{i}^{\prime} \backslash s_{i}} c_{e}\left(x_{e}+w_{i}\right)+\sum_{e \in s_{i}^{\prime} \cap s_{i}} c_{e}\left(x_{e}\right)\right)-\left(\sum_{e \in s_{i} \backslash s_{i}^{\prime}} c_{e}\left(x_{e}\right)+\sum_{e \in s_{i}^{\prime} \cap s_{i}} c_{e}\left(x_{e}\right)\right)\right] \\
& =w_{i}\left[\alpha C_{i}\left(s_{i}^{\prime}, s_{-i}\right)-C_{i}(s)\right] .
\end{aligned}
$$

The first inequality holds due to (3); the second due to the definition of $\alpha$; and the third one because $\alpha \geq 1$. The fact that the cost functions are nonnegative is a critical component in all of them as well. The chain of inequalities above demonstrate that, if $\Phi\left(s_{i}^{\prime}, s_{-i}\right)-\Phi(s)$ is nonnegative then $\alpha C_{i}\left(s_{i}^{\prime}, s_{-i}\right)-C_{i}(s)$ is nonnegative, thus proving (5).

For (6) next, denote $N_{e}=N_{e}(s), x_{e}=x_{e}(s)$ and $N_{e}^{\prime}=N_{e}\left(s^{\prime}\right), x_{e}^{\prime}=x_{e}\left(s^{\prime}\right)$. Then, we have:

$$
\begin{aligned}
\Phi\left(s^{\prime}\right)-\Phi(s) & =\sum_{e \in E} \frac{1}{\alpha_{1, e}} \phi_{e}\left(N_{e}^{\prime}\right)-\sum_{e \in E} \frac{1}{\alpha_{1, e}} \phi_{e}\left(N_{e}\right) \\
& \leq \sum_{e \in E} \frac{\beta_{2, e}}{\alpha_{1, e}} x_{e}^{\prime} c_{e}\left(x_{e}^{\prime}\right)-\sum_{e \in E} \frac{\beta_{1, e}}{\alpha_{1, e}} x_{e} c_{e}\left(x_{e}\right)
\end{aligned}
$$




$$
\begin{aligned}
& \leq \max _{e \in E} \frac{\beta_{2, e}}{\alpha_{1, e}} \cdot \sum_{e \in E} x_{e}^{\prime} c_{e}\left(x_{e}^{\prime}\right)-\min _{e \in E} \frac{\beta_{1, e}}{\alpha_{1, e}} \cdot \sum_{e \in E} x_{e} c_{e}\left(x_{e}\right) \\
& =\max _{e \in E} \frac{\beta_{2, e}}{\alpha_{1, e}} \cdot C\left(s^{\prime}\right)-\min _{e \in E} \frac{\beta_{1, e}}{\alpha_{1, e}} \cdot C(s) \\
& =\min _{e \in E}\left(\beta_{1, e} / \alpha_{1, e}\right) \cdot\left[\beta C\left(s^{\prime}\right)-C(s)\right],
\end{aligned}
$$

where for the first inequality we deployed (4). The chain of inequalities above demonstrate that if $\Phi\left(s^{\prime}\right)-\Phi(s)$ is nonnegative then $\beta C\left(s^{\prime}\right)-C(s)$ is nonnegative as well, this establishing (6).

We continue with defining a critical notion that will act as the medium to utilize our main blackbox tool in Theorem 1. It involves a set of parameters, that determine how "well" a given cost function behaves with respect to two specific, simple analytic properties (namely (7) and (8)). These properties can be interpreted as bounds on the average of the cost function over continuous intervals.

Definition 2 (Good Cost Functions). Fix a congestion game $\mathcal{G}$. A function $c: \mathbb{R}_{+} \longrightarrow \mathbb{R}_{+}$will be called $\left(\alpha_{1}, \alpha_{2}, \beta_{1}, \beta_{2}\right)$-good (with respect to $\mathcal{G}$ ), for $\alpha_{1}, \alpha_{2}, \beta_{1}, \beta_{2}>0$, if there exists a nonnegative constant $\xi$ such that, for all $x \in\{0\} \cup\left[w_{\min }, W\right], w \in\left[w_{\min }, w_{\max }\right]$ :

$$
\alpha_{1} \cdot c(x+w)-\xi \cdot c(w) \leq \frac{1}{w} \int_{x}^{x+w} c(t) d t \leq \alpha_{2} \cdot c(x+w)-\xi \cdot c(w)
$$

and for all $x \in\left[w_{\min }, W\right]$ :

$$
\beta_{1} \cdot c(x)-\xi \cdot c_{\min }(x) \leq \frac{1}{x} \int_{0}^{x} c(t) d t \leq \beta_{2} \cdot c(x)-\xi \cdot c_{\max }(x),
$$

where $c_{\min }(x)=\min _{y \in\left[w_{\min }, x\right]} c(y), c_{\max }(x)=\max _{y \in\left[w_{\min }, x\right]} c(y)$.

Definition 3 (Good Games). A congestion game will be called $\left\{\left(\alpha_{1, j}, \alpha_{2, j}, \beta_{1, j}, \beta_{2, j}\right)\right\}_{j \in J}$-good if any cost function is a conical combination of such good functions. Formally, for any $e \in E$ there exists a nonempty $J_{e} \subseteq J$ and nonnegative constants $\left\{\lambda_{e, j}\right\}_{j \in J_{e}}$, such that

$$
c_{e}(t)=\sum_{j \in J_{e}} \lambda_{e, j} c_{j}(t)
$$

where, for all $j \in J, c_{j}$ is a $\left(\alpha_{1, j}, \alpha_{2, j}, \beta_{1, j}, \beta_{2, j}\right)$-good function (see Definition 2).

Remark 1. Notice that an important special case of Definition 3 is when $J=E, J_{e}=\{e\}$, and $\lambda_{e, e}=1$, meaning that the actual cost functions of the game are good themselves. As a matter of fact, it is not hard to see that any good game $\mathcal{G}$ can be transformed to a strategically equivalent one $\mathcal{G}^{\prime}$ that has that property. First, replace each resource $e$ of $\mathcal{G}$ with a gadget of "parallel" resources $\left\{(e, j) \mid j \in J_{e}\right\}$, each having a cost function of $c_{(e, j)}(t)=\lambda_{e, j} c_{j}(t)$; this results in a strategically equivalent game $\mathcal{G}^{\prime}$ with resources $E^{\prime}=\left\{(e, j) \mid e \in E, j \in J_{e}\right\}$. Next, just observe that Definition 2 is invariant under nonnegative scalar multiplication: since functions $c_{j}$ satisfy conditions (7) and (8), so do functions $\lambda_{e, j} \cdot c_{j}$ that are exactly the cost functions of the new game $\mathcal{G}^{\prime}$.

Remark 2 (Increasing Good Functions). If a cost function is nondecreasing, then (8) can be replaced by the (stronger, sufficient) condition:

$$
\beta_{1} c(x) \leq \frac{1}{x} \int_{0}^{x} c(t) d t \leq\left(\beta_{2}-\xi\right) c(x),
$$

since $0 \leq c(y) \leq c(x)$ for any $y \in\left[w_{\min }, x\right]$.

Now we are ready to state our main tool. This is essentially the interface of our entire framework: under the hood it uses a specific potential function form (see (9)), but its statement involves only the goodness parameters of the cost functions, as defined above. In that way, one can readily derive meaningful bounds about the existence of $(\alpha, \beta)$-equilibria in a black-box way, just by studying the simple analytic properties given in (2) and the plugging the parameters in the theorem below: 
Theorem 1. Any $\left\{\left(\alpha_{1, j}, \alpha_{2, j}, \beta_{1, j}, \beta_{2, j}\right)\right\}_{j \in J}$-good congestion game has an $(\alpha, \beta)$-equilibrium with

$$
\alpha=\max _{j \in J} \frac{\alpha_{2, j}}{\alpha_{1, j}} \quad \text { and } \quad \beta=\frac{\max _{j \in J} \beta_{2, j} / \alpha_{1, j}}{\min _{j \in J} \beta_{1, j} / \alpha_{1, j}} .
$$

Proof. First notice that, by Remark 1, it is without loss to assume that $J=E$ and that any cost function $c_{e}, e \in E$, is $\left(\alpha_{1, e}, \alpha_{2, e}, \beta_{1, e}, \beta_{2, e}\right)$-good. Denote by $\xi_{e}$ (a choice of) the parameter $\xi$ for which resource $e$ satisfies Definition 2.

We will then show that functions

$$
\phi_{e}(I)=\int_{0}^{w_{I}} c_{e}(t) d t+\xi_{e} \sum_{i \in I} w_{i} c_{e}\left(w_{i}\right)
$$

satisfy the conditions of Lemma 1 ,

Fix some resource $e \in E$, a player $i$ and a subset $I \subseteq N \backslash\{i\}$ of remaining players. For simplicity, from now on we drop the $e$ subscripts and also denote $w=w_{i}$ and $x=w_{I}$. Then,

$$
\begin{aligned}
\phi(I \cup\{i\})-\phi(I) & =\int_{0}^{x+w} c_{e}(t) d t-\int_{0}^{x} c_{e}(t) d t+\xi_{e}\left(\sum_{j \in I} w_{j} c_{e}\left(w_{j}\right)-\sum_{j \in I \cup\{i\}} w_{j} c_{e}\left(w_{j}\right)\right) \\
& =\int_{x}^{x+w} c(t) d t+\xi w c(w) .
\end{aligned}
$$

So, by deploying (7), it is not difficult to see that

$$
\alpha_{1} c(x+w) \leq \frac{1}{w}[\phi(I \cup\{i\})-\phi(I)] \leq \alpha_{2} c(x+w),
$$

and thus condition (3) of Lemma 1 is indeed satisfied.

Next, observe that since $w_{j} \in\left[w_{\min }, w_{\max }\right]$ for all $j \in I$, and $x=\sum_{j \in I} w_{j}$, we have the bounds

$$
c_{\min }(x) \leq \min _{j \in I} c\left(w_{j}\right) \leq \frac{1}{x} \sum_{j \in I} w_{j} c\left(w_{j}\right) \leq \max _{j \in I} c\left(w_{j}\right) \leq c_{\max }(x),
$$

where the first and the last inequalities hold due to the fact that $\left\{w_{j} \mid j \in I\right\} \subseteq\left[w_{\min }, x\right]$. Assuming $I \neq \emptyset$, we have that $x \in\left[w_{\min }, W\right]$ and so we can use (10) and (8) to bound $\frac{1}{x} \phi(I)$ from below and above by:

$$
\beta_{1} c(x) \leq \frac{1}{x} \phi(I)=\frac{1}{x} \int_{0}^{x} c(t) d t+\xi \frac{1}{x} \sum_{j \in I} w_{j} c\left(w_{j}\right) \leq \beta_{2} c(x) .
$$

Thus, condition (4) of Lemma 1 is also satisfied.

\section{Applications}

In this section we present several applications of our black-box Theorem 1, that demonstrate both its power and simplicity. In accordance to the nature of that tool, they all share a common structure: first, we prove lemmas describing the right goodness parameters (according to Definition 2) for each special cost function of interest (see Lemmas 2 to 5); then, we plug them in Theorem 1 to derive our bounds (see Theorems 2 to 4 ). 


\subsection{Polynomial Costs}

We start with polynomial cost functions, arguably the most studied setting in congestion games. We recover the result from Caragiannis and Fanelli [CF19] that, for polynomials of degree at most $d$ with nonnegative coefficients, there exist $(d+\delta)$-approximate equilibria with social cost at most $\frac{d+1}{d+\delta}$ times the optimum, for any $\delta \in[0,1]$. This is the currently best known guarantee of $(\alpha, \beta)$-equilibria for polynomial cost functions. Let us begin by analysing the goodness parameters of each monomial.

Lemma 2. Any monomial of degree $d \geq 1$ is $\left(\mu, 1, \frac{1}{d+1}, \mu\right)$-good, for any $\mu \in\left[\frac{1}{d+1}, \frac{1}{d}\right]$.

Proof. Fix a degree $d \geq 1$. We will show that the function $c(x)=x^{d}$ satisfies conditions (7) and (8) with

$$
\alpha_{1}=\xi+\frac{1}{d+1}, \quad \alpha_{2}=1, \quad \beta_{1}=\frac{1}{d+1}, \quad \beta_{2}=\xi+\frac{1}{d+1},
$$

for all $\xi \in\left[0, \frac{1}{d(d+1)}\right]$. Then, performing the change of variables $\mu=\xi+\frac{1}{d+1}$ establishes our lemma, since $\mu \in\left[0+\frac{1}{d+1}, \frac{1}{d(d+1)}+\frac{1}{d+1}\right]=\left[\frac{1}{d+1}, \frac{1}{d}\right]$.

To prove the bounds in $\alpha_{1}, \alpha_{2}$, we are interested in the quantity

$$
a(w, x)=\frac{1}{w} \int_{x}^{x+w} c(t) d t+\xi c(w)=\frac{1}{(d+1) w}\left((x+w)^{d+1}-x^{d+1}\right)+\xi w^{d} .
$$

By applying the binomial expansion rules, and collecting similar terms, we can further write

$$
\begin{aligned}
a(w, x) & =\frac{1}{(d+1) w}\left(w^{d+1}+\sum_{j=1}^{d}\left(\begin{array}{c}
d+1 \\
j
\end{array}\right) x^{j} w^{d+1-j}+x^{d+1}-x^{d+1}\right)+\xi w^{d+1} \\
& =\frac{1}{d+1}\left(w^{d}+\sum_{j=1}^{d}\left(\begin{array}{c}
d+1 \\
j
\end{array}\right) x^{j} w^{d-j}\right)+\xi w^{d} \\
& =\left(\frac{1}{d+1}+\xi\right) w^{d}+\sum_{j=1}^{d} \frac{1}{d+1}\left(\begin{array}{c}
d+1 \\
j
\end{array}\right) x^{j} w^{d-j} \\
& =\left(\xi+\frac{1}{d+1}\right) w^{d}+\sum_{j=1}^{d} \frac{1}{d-j+1}\left(\begin{array}{l}
d \\
j
\end{array}\right) x^{j} w^{d-j}
\end{aligned}
$$

where in the last step we simply use the fact that, for $1 \leq j \leq d, \frac{1}{d+1}\left(\begin{array}{c}d+1 \\ j\end{array}\right)=\frac{1}{d-j+1}\left(\begin{array}{c}d \\ j\end{array}\right)$. We would like to get upper and lower bounds on $a(w, x)$ involving $c(x+w)$, which can be written as

$$
c(x+w)=(x+w)^{d}=w^{d}+\sum_{j=1}^{d}\left(\begin{array}{l}
d \\
j
\end{array}\right) x^{j} w^{d-j} .
$$

By comparing the coefficients of (11) and (12), we get that (7) is satisfied with

$$
\begin{aligned}
& \alpha_{1}=\min \left\{\xi+\frac{1}{d+1}, \min _{j=1, \ldots, d}\left\{\frac{1}{d-j+1}\right\}\right\}=\min \left\{\xi+\frac{1}{d+1}, \frac{1}{d}\right\}=\xi+\frac{1}{d+1} \\
& \alpha_{2}=\max \left\{\xi+\frac{1}{d+1}, \max _{j=1, \ldots, d}\left\{\frac{1}{d-j+1}\right\}\right\}=\max \left\{\xi+\frac{1}{d+1}, 1\right\}=1,
\end{aligned}
$$

where to compute the maxima and minima we used the fact that $\xi+\frac{1}{d+1} \leq \frac{1}{d(d+1)}+\frac{1}{d+1}=\frac{1}{d} \leq 1$, due to the assumptions that $\xi \leq \frac{1}{d(d+1)}$ and $d \geq 1$.

For the bounds in $\beta_{1}, \beta_{2}$, since $x^{d}$ is nondecreasing we can use the simpler condition ( $\left(8^{\prime}\right)$. Then, we only have to observe that

$$
\frac{1}{x} \int_{0}^{x} c(t) d t=\frac{1}{d+1} x^{d}=\frac{1}{d+1} c(x)
$$


and

$$
\frac{1}{x} \int_{0}^{x} c(t) d t+\xi c(x)=\frac{1}{d+1} x^{d}+\xi x^{d}=\left(\xi+\frac{1}{d+1}\right) c(x)
$$

For the special case of constant cost functions, i.e., 0 -degree monomials, it is not difficult to get the following:

Lemma 3. Any constant function is $(1,1,1,1)$-good.

Proof. Follows directly from Definition 2 by taking $\xi=0$ : for any constant function $c(x)=c$ we have

$$
c(x+w)=c(x)=\frac{1}{w} \int_{x}^{x+w} c(t) d t=\frac{1}{x} \int_{0}^{x} c(t) d t=c .
$$

Theorem 2. Any weighted polynomial congestion game of degree $d \geq 1$ has an $\left(\lambda, \frac{d+1}{\lambda}\right)$-equilibrium, for any $\lambda \in[d, d+1]$.

Proof. Fix a maximum degree $d \geq 1$ and a parameter $\lambda \in[d, d+1]$. Utilizing Lemma 2 with $\mu=\frac{1}{k+1}$ and Lemma 3, we can see that monomials of degree $k=0, \ldots, d-1$ are $\left(\frac{1}{k+1}, 1, \frac{1}{k+1}, \frac{1}{k+1}\right)$-good; and utilizing Lemma 2 with $\mu=\frac{1}{\lambda}$ we get that the monomial of degree $d$ is $\left(\frac{1}{\lambda}, 1, \frac{1}{d+1}, \frac{1}{\lambda}\right)$-good.

Since any polynomial of degree (at most) $d$ is a conical combination of monomials of degree $k=$ $0,1, \ldots, d$, in light of Definition 3, we can deduce that our game is $\left\{\left(\alpha_{1, k}, \alpha_{2, k}, \beta_{1, k}, \beta_{2, k}\right)\right\}_{k=0, \ldots, d}$-good, with

$$
\alpha_{1, k}=\left\{\begin{array}{ll}
\frac{1}{\lambda}, & k=d, \\
\frac{1}{k+1}, & k<d ;
\end{array} \quad \alpha_{2, k}=1 ; \quad \beta_{1, k}=\left\{\begin{array}{ll}
\frac{1}{d+1}, & k=d, \\
\frac{1}{k+1}, & k<d ;
\end{array} \quad \beta_{2, k}= \begin{cases}\frac{1}{\lambda}, & k=d, \\
\frac{1}{k+1}, & k<d .\end{cases}\right.\right.
$$

Thus, by Theorem 1 we conclude that our game has an $(\alpha, \beta)$-equilibrium with

$$
\alpha=\max _{0 \leq k \leq d} \frac{\alpha_{2, k}}{\alpha_{1, k}}=\max \{1,2, \ldots, d, \lambda\}=\lambda,
$$

and

$$
\beta=\frac{\max _{0 \leq k \leq d} \frac{\beta_{2, k}}{\alpha_{1, k}}}{\min _{0 \leq k \leq d} \frac{\beta_{1, k}}{\alpha_{1, k}}}=\frac{1}{\min \left\{1, \ldots, 1, \frac{1 /(d+1)}{1 / \lambda}\right\}}=\max \left\{1, \frac{d+1}{\lambda}\right\}=\frac{d+1}{\lambda} .
$$

The parameter $\lambda$ quantifies the trade-off curve between the approximation guarantee on the existence of $\alpha$-approximate equilibria and their PoS. At one extreme case $\lambda=d+1$, we get that $\alpha=d+1$ and $\beta=1$; in other words, there always exist $(d+1)$-approximate equilibria with an optimal PoS of 1 (as a matter of fact, from [CGGS19] we already know that every social optimum is itself a $(d+1)$ approximate equilibrium). At the other extreme case $\lambda=d$, we get that

$$
\alpha=d, \quad \beta=\frac{d+1}{d}=1+\frac{1}{d}
$$

in other words, there always exist $d$-approximate equilibria with PoS at most $1+\frac{1}{d}$. 


\subsection{Concave Costs}

We now look at nondecreasing concave cost functions. The best known result in this setting is due to Hansknecht et al. [HKS14], who state that 3/2-approximate equilibria exist. However, the proof in their paper is not complete. Moreover, the PoS of the existing approximate equilibria is not discussed. In this section, not only we provide a simpler proof of this result, but we also extend it for a range of $\lambda$-approximate equilibria with $\lambda \in[3 / 2,2]$, and for a guarantee on the PoS.

Lemma 4. Any nondecreasing concave function is $\left(\mu, \mu+\frac{1}{2}, \frac{1}{2}, \mu+\frac{1}{2}\right)$-good, for all $\mu \in\left[\frac{1}{2}, 1\right]$.

Proof. Fix a nondecreasing concave function $c: \mathbb{R}_{+} \longrightarrow \mathbb{R}_{+}$and a parameter $0 \leq \xi \leq \frac{1}{2}$. First note that, since $c$ is nonnegative and concave, it must be subadditive. That is, for all $x, z \geq 0$ :

$$
c(x)+c(z) \geq c(x+z)
$$

Furthermore, from the Hermite-Hadamard inequality (see, e.g., [ML85]) and the fact that $c$ is nondecreasing, for any $0 \leq a<b$ :

$$
\frac{f(a)+f(b)}{2} \leq \frac{1}{b-a} \int_{a}^{b} f(t) d t \leq f(b) .
$$

Applying first (14) for $a=x$ and $b=x+w$ we get that

$$
\frac{c(x)+c(x+w)}{2} \leq \frac{1}{w} \int_{x}^{x+w} c(t) d t \leq c(x+w),
$$

so

$$
\frac{1}{w} \int_{x}^{x+w} c(t) d t+\xi \cdot c(w) \leq c(x+w)+\xi c(w) \leq(1+\xi) c(x+z)
$$

and

$$
\begin{array}{rlrl}
\frac{1}{w} \int_{x}^{x+w} c(t) d t+\xi \cdot c(w) & \geq \frac{c(x)+c(x+w)}{2}+\xi c(w) & \\
& \geq \frac{1}{2} c(x+w)+\xi[c(x)+c(w)], & & \text { since } \xi \leq \frac{1}{2} \\
& \geq\left(\frac{1}{2}+\xi\right) c(x+w), & & \text { due to (13). }
\end{array}
$$

Thus, condition (7) is satisfied with $\alpha_{1}=\frac{1}{2}+\xi, \alpha_{2}=1+\xi$.

Next, applying (14) for $a=0$ and $b=x$ we get that

$$
\frac{1}{2} c(x) \leq \frac{c(0)+c(x)}{2} \leq \frac{1}{x} \int_{0}^{x} c(t) d t \leq c(x)=(1+\xi) c(x)-\xi c(x)
$$

thus condition ( $\left.8^{\prime}\right)$ is satisfied with $\beta_{1}=\frac{1}{2}, \beta_{2}=1+\xi$.

Summarizing, we have shown (see Definition 2) that any concave cost function is $\left(\xi+\frac{1}{2}, \xi+1, \frac{1}{2}, \xi+1\right)$ good, for any $\xi \in\left[0, \frac{1}{2}\right]$. Performing the change of variables $\mu=\xi+\frac{1}{2}$ concludes our proof.

Theorem 3. Any weighted congestion game with nondecreasing concave cost functions has $a\left(\lambda, \frac{\lambda}{\lambda-1}\right)$ equilibrium, for any $\lambda \in\left[\frac{3}{2}, 2\right]$.

Proof. Fix a parameter $\lambda \in\left[\frac{3}{2}, 2\right]$ and let $\mu=\frac{1}{2(\lambda-1)}$. Then, $\mu \in\left[\frac{1}{2}, 1\right]$ and thus, due to Lemma 4, we can deduce that our game is $\left(\mu, \mu+\frac{1}{2}, \frac{1}{2}, \mu+\frac{1}{2}\right)$-good (according to Definition 2). Deploying Theorem 1 we can establish the existence of an a $(\alpha, \beta)$-equilibrium with

$$
\alpha=\frac{\mu+\frac{1}{2}}{\mu}=1+\frac{1}{2 \mu}=1+(\lambda-1)=\lambda \quad \text { and } \quad \beta=\frac{\left(\mu+\frac{1}{2}\right) / \mu}{\frac{1}{2} / \mu}=2 \mu+1=\frac{1}{\lambda-1}+1=\frac{\lambda}{\lambda-1} .
$$




\subsection{Fair Cost Sharing}

In this section, we focus on the fair cost sharing model in which $c_{e}(x)=\frac{a_{e}}{x}$, where $a_{e}$ is a positive, resource-dependent value. We assume that $w_{\min }=1$; this is without loss, since we can just rescale the player weights. This setting was studied by Chen and Roughgarden [CR09]. Here we improve on their results (see Fig. 1), with a simpler proof.

We must notice that the function $x \mapsto a_{e} / x$ is not integrable in an interval starting at 0 , and hence we cannot immediately apply our Definition 2 . However, based on our discussion in Section 2.1 we can modify the game in order to overcome this. First, we assume for our analysis that $a_{e}=1$ since any other choice of $a_{e}$ can be seen as a trivial conical combination of the function $1 / x$ (see Definition 3 ). Next, we change the cost function $c_{e}(x)$ to be constant and equal to $\lambda$ in the interval $[0,1)$, for some $\lambda \geq 1$.

Lemma 5. Fix a weighted congestion game with $w_{\min }=1$. For any $\lambda \geq 1$, the cost function

$$
c(x)= \begin{cases}1 / x, & x \geq 1 \\ \lambda, & 0 \leq x<1\end{cases}
$$

is $\left(\alpha_{1}, \alpha_{2}, \beta_{1}, \beta_{2}\right)$-good with

$$
\begin{array}{ll}
\alpha_{1}=1, & \alpha_{2}=\max \left(\left(1+\frac{1}{w_{\max }}\right) \ln \left(1+w_{\max }\right), \ln \left(w_{\max }\right)+\lambda\right), \\
\beta_{1}=\lambda, & \beta_{2}=\ln W+\lambda .
\end{array}
$$

Proof. We will choose $\xi=0$ in the Definition 2 of good cost functions. Thus, we need to find nonnegative quantities $\alpha_{1}, \alpha_{2}, \beta_{1}, \beta_{2}$ such that, for $x \in\{0\} \cup[1, W], w \in\left[1, w_{\max }\right]$,

$$
\alpha_{1} \cdot c(x+w) \leq \frac{1}{w} \int_{x}^{x+w} c(t) d t \leq \alpha_{2} \cdot c(x+w)
$$

and for all $x \in[1, W]$,

$$
\beta_{1} \cdot c(x) \leq \frac{1}{x} \int_{0}^{x} c(t) d t \leq \beta_{2} \cdot c(x)
$$

For the bounds in $\alpha_{1}, \alpha_{2}$, we are interested in upper and lower bounds on the ratio

$$
R(w, x)=\frac{\frac{1}{w} \int_{x}^{x+w} c(t) d t}{c(x+w)} .
$$

When $x \geq 1$, this becomes

$$
R(w, x)=\frac{\frac{1}{w}(\ln (x+w)-\ln (x))}{\frac{1}{x+w}}=\left(1+\frac{x}{w}\right) \ln \left(1+\frac{w}{x}\right) ;
$$

on the other hand, when $x=0$, this becomes

$$
R(w, 0)=\frac{\frac{1}{w}(\ln (w)+\lambda)}{\frac{1}{w}}=\ln (w)+\lambda .
$$

Thus, we get

$$
R(w, x)=\left\{\begin{array}{cc}
\left(1+\frac{x}{w}\right) \ln \left(1+\frac{w}{x}\right), & x \geq 1 \\
\ln w+\lambda, & x=0 .
\end{array}\right.
$$

In Lemma 6 in the Appendix, we show that the upper branch of $R(w, x)$ is increasing in $w$ and decreasing in $x$; hence, it is maximized at $w \rightarrow w_{\max }, x \rightarrow 1$, for a value of $R\left(w_{\max }, 1\right)=\left(1+\frac{1}{w_{\max }}\right) \ln (1+$ $\left.w_{\max }\right)$; and minimized at $w \rightarrow 1, x \rightarrow W$, for a value of $R(1, W) \geq R(1, \infty)=1$. On the other hand, the 

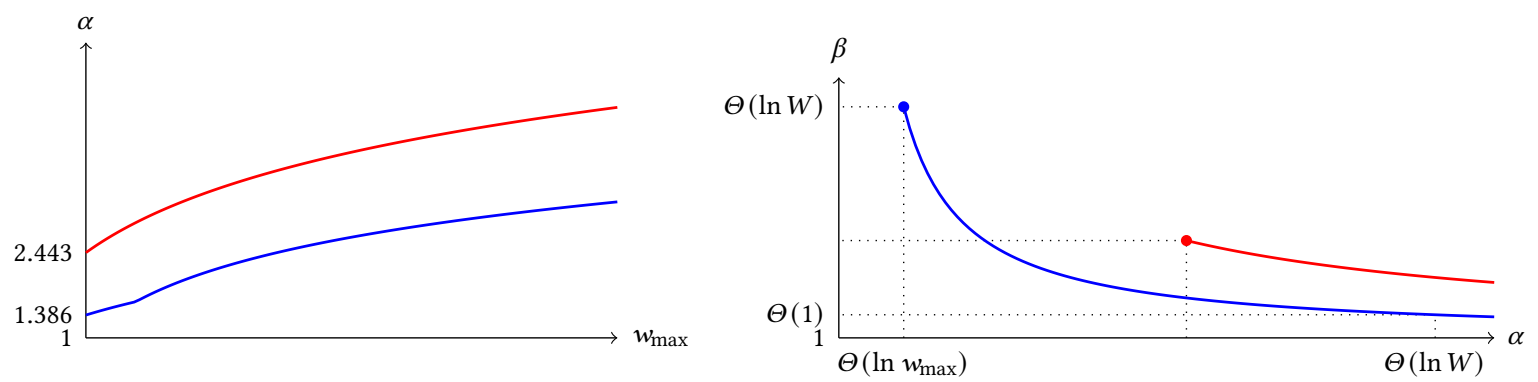

Figure 1: Fair cost sharing games. Left: guarantee on the existence of $\alpha$-approximate equilibria, as a function of $w_{\max }$, given by Theorem 4 (setting $\lambda=1$ ). Right: trade-off curve for the existence of $(\alpha, \beta)$-equilibria, given by Theorem 4 ; here we choose $w_{\max }=3, W=50$. For comparison, the previously best bounds [CR09, Theorem 5.1 and Lemma 5.3] are plotted in red, while our results are in blue. The fact that the blue line of the right plot starts earlier is a direct consequence of our results providing a strictly better (smaller) absolute existence guarantee $\alpha$ (see left plot).

lower branch is maximized at $w \rightarrow w_{\max }$, for a value of $R\left(w_{\max }, 0\right)=\ln \left(w_{\max }\right)+\lambda$; and minimized at $w \rightarrow 1$, for a value of $R(1,0)=\lambda \geq 1$. This gives the desired bounds on $\alpha_{1}$ and $\alpha_{2}$.

Next, we look at the bounds in $\beta_{1}, \beta_{2}$. Since $x \in[1, W]$ we have that $\int_{0}^{x} c(t) d t=\ln x+\lambda$. Moreover, it is immediate to observe that

$$
\lambda \cdot c(x)=\lambda \cdot \frac{1}{x} \leq \frac{1}{x}(\ln x+\lambda) \leq \frac{1}{x}(\ln W+\lambda)=(\ln W+\lambda) \cdot c(x) .
$$

Theorem 4. Fix a fair cost sharing game with unit minimum weight $\left(w_{\min }=1\right)$, and let $w_{\max }, W$ be the maximum weight and the maximum total load. Then, for any $\lambda \geq 1$, our game has an $(\alpha, \beta)$-equilibrium where

$$
\alpha=\max \left(\left(1+\frac{1}{w_{\max }}\right) \ln \left(1+w_{\max }\right), \ln \left(w_{\max }\right)+\lambda\right), \quad \beta=1+\frac{\ln W}{\lambda} .
$$

Proof. Combining Theorem 1 with Lemma 5 we conclude that, for $\lambda \geq 1$, our game has an $(\alpha, \beta)$ equilibrium with

$$
\alpha=\frac{\alpha_{2}}{\alpha_{1}}=\max \left(\left(1+\frac{1}{w_{\max }}\right) \ln \left(1+w_{\max }\right), \ln \left(w_{\max }\right)+\lambda\right), \quad \beta=\frac{\beta_{2} / \alpha_{1}}{\beta_{1} / \alpha_{1}}=\frac{\ln W+\lambda}{\lambda}=1+\frac{\ln W}{\lambda} .
$$

The parameter $\lambda$ quantifies the trade-off curve between the approximation guarantee on equilibria and their price of stability. At one extreme case $\lambda=1$, we get that

$$
\alpha=\max \left(\left(1+\frac{1}{w_{\max }}\right) \ln \left(1+w_{\max }\right), \ln \left(w_{\max }\right)+1\right)=\Theta\left(\ln w_{\max }\right), \quad \beta=1+\ln W ;
$$

in other words, there exist $\Theta\left(\ln w_{\max }\right)$-approximate equilibria with price of stability $\Theta(\ln W)$. At the other extreme case $\lambda=\Theta(\ln W)$, we get that

$$
\alpha=\max \left(\left(1+\frac{1}{w_{\max }}\right) \ln \left(1+w_{\max }\right), \ln \left(w_{\max }\right)+\Theta(\ln W)\right)=\Theta(\ln W), \quad \beta=1+\frac{\ln W}{\Theta(\ln W)}=\Theta(1) ;
$$

in other words, there exist $\Theta(\ln W)$-approximate equilibria with constant price of stability $\Theta(1)$. The complete trade-off curve can be seen in Fig. 1 (right). We can also compare our results with the best known upper bounds. In [CR09, Lemma 5.3], it was shown that $\alpha$-approximate equilibria exist for $\alpha \geq \log _{2}\left[e\left(1+w_{\max }\right)\right]$; and in [CR09, Theorem 5.1], it was shown that $\left(f, 1+\frac{2 \log _{2}(1+W)}{f}\right)$-equilibria exist for any $f \geq 2 \log _{2}\left[e\left(1+w_{\max }\right)\right]$. As Fig. 1 shows, we improve on both results. 


\subsection{Mixtures of Cost Functions}

A big advantage of our approach is that we can study the existence of $(\alpha, \beta)$-equilibria for games that merge cost functions of two or more different types. For example, in this section we look at congestion games that have both concave costs and polynomial costs (as well as any conical combination). Interestingly, we show that this results in only a small increase in the PoS guarantee of Theorem 2, while the existence guarantee stays the same. For the following theorem we consider polynomials of degree at least 2 , since affine functions are themselves concave and would be already captured by Theorem 3 .

Theorem 5. Any weighted congestion game with cost functions that are conical combinations of concave and polynomial costs of maximum degree $d \geq 2$ has an $\left(\lambda, 1+\frac{d+1}{\lambda}\right)$-equilibrium, for any $\lambda \in[d, d+1]$.

Proof. Fix a maximum degree $d \geq 2$ for the polynomial costs and a parameter $\lambda \in[d, d+1]$. By defining $\mu=\frac{d+1}{2 \lambda}$ we have that $\frac{1}{2} \leq \mu \leq \frac{1}{2}\left(1+\frac{1}{d}\right) \leq 1$, and so by applying Lemma 4 we can derive that any concave cost is $\left(\mu, \mu+\frac{1}{2}, \frac{1}{2}, \mu+\frac{1}{2}\right)$-good. Next, by Lemmas 2 and 3 we can derive that all monomials of degree $k=0, \ldots, d-1$ are $\left(\frac{1}{k+1}, 1, \frac{1}{k+1}, \frac{1}{k+1}\right)$-good and the monomial of degree $d$ is $\left(\frac{1}{\lambda}, 1, \frac{1}{d+1}, \frac{1}{\lambda}\right)$-good.

Deploying our black-box tool Theorem 1 (and shortcutting some calculations that we have already performed in the proof of Theorem 2) we can guarantee the existence of an $(\alpha, \beta)$-equilibrium with

$$
\alpha=\max \left\{1+\frac{1}{2 \mu}, \lambda\right\}=\max \left\{1+\frac{\lambda}{d+1}, \lambda\right\}=\lambda,
$$

since $2 \leq d \leq \lambda \leq d+1$, and

$$
\beta=\frac{\max \left\{\frac{\mu+1 / 2}{\mu}, 1\right\}}{\min \left\{\frac{1 / 2}{\mu}, \frac{\lambda}{d+1}\right\}}=\frac{1+\frac{1}{2 \mu}}{\min \left\{\frac{1}{2 \mu}, \frac{\lambda}{d+1}\right\}}=1+2 \mu=1+\frac{d+1}{\lambda},
$$

where for the third equality we used that, from the definition of $\mu, \frac{1}{2 \mu}=\frac{\lambda}{d+1}$.

\section{Acknowledgements}

We thank Martin Gairing for interesting discussions.

\section{References}

[ADK $\left.{ }^{+} 08\right]$ Elliot Anshelevich, Anirban Dasgupta, Jon Kleinberg, Éva Tardos, Tom Wexler, and Tim Roughgarden. The price of stability for network design with fair cost allocation. SIAM fournal on Computing, 38(4):1602-1623, January 2008. doi:10.1137/070680096.

[BCFM13] Vittorio Bilò, Ioannis Caragiannis, Angelo Fanelli, and Gianpiero Monaco. Improved lower bounds on the price of stability of undirected network design games. Theory of Computing Systems, 52(4):668-686, May 2013. doi:10.1007/s00224-012-9411-6.

[BFM14] Vittorio Bilò, Michele Flammini, and Luca Moscardelli. The price of stability for undirected broadcast network design with fair cost allocation is constant. Games and Economic Behavior, 2014. doi:10.1016/j.geb.2014.09.010.

[CF19] Ioannis Caragiannis and Angelo Fanelli. On approximate pure Nash equilibria in weighted congestion games with polynomial latencies. In Proceedings of the 46th International Colloquium on Automata, Languages, and Programming (ICALP), pages 133:1-133:12, 2019. doi: 10.4230/LIPICs. ICALP. 2019.133.

[CFGS11] Ioannis Caragiannis, Angelo Fanelli, Nick Gravin, and Alexander Skopalik. Efficient computation of approximate pure Nash equilibria in congestion games. In Proceedings of the 52nd IEEE Annual Symposium on Foundations of Computer Science (FOCS), pages 532-541, 2011. doi:10.1109/focs.2011.50. 
[CGG $\left.{ }^{+} 20\right]$ George Christodoulou, Martin Gairing, Yiannis Giannakopoulos, Diogo Poças, and Clara Waldmann. Existence and complexity of approximate equilibria in weighted congestion games. In Proceedings of the 47th International Colloquium on Automata, Languages, and Programming (ICALP), pages 32:1-32:18, 2020. doi:10 .4230/LIPICs. ICALP . 2020 . 32.

[CGGS19] George Christodoulou, Martin Gairing, Yiannis Giannakopoulos, and Paul G. Spirakis. The price of stability of weighted congestion games. SIAM fournal on Computing, 48(5):15441582, 2019. doi:10.1137/18M1207880.

[CKS11] George Christodoulou, Elias Koutsoupias, and Paul G. Spirakis. On the performance of approximate equilibria in congestion games. Algorithmica, 61(1):116-140, 2011. doi:10.1007/s00453-010-9449-2.

[CR09] Ho-Lin Chen and Tim Roughgarden. Network design with weighted players. Theory of Computing Systems, 45(2):302-324, July 2009. doi : 10 .1007/s00224-008-9128-8.

[CSSM04] José R. Correa, Andreas S. Schulz, and Nicolás E. Stier-Moses. Selfish routing in capacitated networks. Mathematics of Operations Research, 29(4):961-976, 2004. doi:10.1287/moor.1040.0098.

[DS08] Juliane Dunkel and Andreas S. Schulz. On the complexity of pure-strategy Nash equilibria in congestion and local-effect games. Mathematics of Operations Research, 33(4):851-868, 2008. doi:10.1287/moor.1080.0322.

[FHP16] Rupert Freeman, Samuel Haney, and Debmalya Panigrahi. On the Price of Stability of Undirected Multicast Games, pages 354-368. 2016. doi:10.1007/978-3-662-54110-4_25.

[FKK ${ }^{+}$09] Dimitris Fotakis, Spyros Kontogiannis, Elias Koutsoupias, Marios Mavronicolas, and Paul Spirakis. The structure and complexity of Nash equilibria for a selfish routing game. Theoretical Computer Science, 410(36):3305-3326, 2009. do i:10 .1016/ j . tcs.2008.01.004.

[FKL $\left.{ }^{+} 06\right]$ Amos Fiat, Haim Kaplan, Meital Levy, Svetlana Olonetsky, and Ronen Shabo. On the price of stability for designing undirected networks with fair cost allocations. In Proceedings of the 33rd International ColloquiumAutomata, Languages and Programming (ICALP), pages 608-618, 2006. doi:10.1007/11786986_53.

[FKS05] Dimitris Fotakis, Spyros Kontogiannis, and Paul Spirakis. Selfish unsplittable flows. Theoretical Computer Science, 348(2):226-239, 2005. doi:10.1016/j .tcs .2005.09.024.

[GMV05] M. Goemans, Vahab Mirrokni, and A. Vetta. Sink equilibria and convergence. In Proceedings of the 46th Annual IEEE Symposium on Foundations of Computer Science (FOCS), pages 142151, 2005. doi:10.1109/SFCS.2005.68.

[GP20] Yiannis Giannakopoulos and Diogo Poças. A unifying approximate potential for weighted congestion games. In Proceedings of the 13th Symposium on Algorithmic Game Theory (SAGT), pages 99-113,2020. doi:10.1007/978-3-030-57980-7_7.

[HK12] Tobias Harks and Max Klimm. On the existence of pure Nash equilibria in weighted congestion games. Mathematics of Operations Research, 37(3):419-436, 2012. doi:10.1287/moor.1120.0543.

[HKM12] Tobias Harks, Max Klimm, and Rolf H Möhring. Strong equilibria in games with the lexicographical improvement property. International fournal of Game Theory, 42(2):461-482, 2012. doi:10.1007/s00182-012-0322-1. 
[HKS14] Christoph Hansknecht, Max Klimm, and Alexander Skopalik. Approximate pure Nash equilibria in weighted congestion games. In Proceedings of the 17th International Workshop on Approximation Algorithms for Combinatorial Optimization Problems (APPROX), pages 242257, 2014. doi:10 .4230/LIPICs . APPROX-RANDOM. 2014. 242.

[KP99] Elias Koutsoupias and Christos Papadimitriou. Worst-case equilibria. In Proceedings of the 16th Annual Symposium on Theoretical Aspects of Computer Science (STACS), pages 404-413, 1999. doi:10.1016/j.cosrev.2009.04.003.

[LO01] Lavy Libman and Ariel Orda. Atomic resource sharing in noncooperative networks. Telecommunication Systems, 17(4):385-409, August 2001. doi:10.1023/A:1016770831869.

[ML85] D. S. Mitrinović and I. B. Lacković. Hermite and convexity. Aequationes mathematicae, 28:229-232, 1985. URL: http://eudml.org/doc/137060.

[MS96] Dov Monderer and Lloyd S. Shapley. Potential games. Games and Economic Behavior, 14(1):124-143, 1996. doi:10.1006/game.1996.0044.

[NRTV07] Noam Nisan, Tim Roughgarden, Éva Tardos, and Vijay Vazirani, editors. Algorithmic Game Theory. Cambridge University Press, 2007. doi:10 .1017/CBO9780511800481.

[Pap01] Christos Papadimitriou. Algorithms, games, and the internet. In Proceedings of the 33rd Annual ACM Symposium on Theory of Computing (STOC), pages 749-753, 2001. doi:10.1145/380752.380883.

[PS07] Panagiota N. Panagopoulou and Paul G. Spirakis. Algorithms for pure Nash equilibria in weighted congestion games. Journal of Experimental Algorithmics, 11:27, February 2007. doi: $10.1145 / 1187436.1216584$.

[Ros73] Robert W. Rosenthal. A class of games possessing pure-strategy Nash equilibria. International fournal of Game Theory, 2(1):65-67, 1973. doi:10 .1007/BF01737559.

[Rou07] Tim Roughgarden. Routing games. In Noam Nisan, Tim Roughgarden, Éva Tardos, and Vijay Vazirani, editors, Algorithmic Game Theory, chapter 18. Cambridge University Press, 2007. doi:10.1017/CBO9780511800481.020.

[Rou16] Tim Roughgarden. Twenty Lectures on Algorithmic Game Theory. Cambridge University Press, 2016. doi:10.1017/cbo9781316779309.

[TW07] Éva Tardos and Tom Wexler. Network formation games and the potential function method. In Noam Nisan, Tim Roughgarden, Éva Tardos, and Vijay Vazirani, editors, Algorithmic Game Theory, chapter 19. Cambridge University Press, 2007. doi:10.1017/cbo9780511800481.021.

[Vöc07] Berthold Vöcking. Selfish load balancing. In Noam Nisan, Tim Roughgarden, Éva Tardos, and Vijay Vazirani, editors, Algorithmic Game Theory, chapter 20. Cambridge University Press, 2007. doi:10.1017/cbo9780511800481.022.

\section{A Technical Lemmas}

Lemma 6. For $w, x \in[1, \infty)$, the function

$$
R(w, x)=\left(1+\frac{x}{w}\right) \ln \left(1+\frac{w}{x}\right)
$$

is increasing in $w$ and decreasing in $x$. 
Proof. Let us apply the change of variables $\frac{1}{z} \equiv 1+\frac{x}{w}$, so that we can write

$$
R(w, x)=\left(1+\frac{x}{w}\right) \ln \left(1+\frac{w}{x}\right) \equiv \frac{\ln \left(\frac{1}{1-z}\right)}{z} .
$$

Since $w, x \in[1, \infty)$, it follows that $1 / z \in(1, \infty)$ and thus $z \in(0,1)$. Notice now that $\ln \left(\frac{1}{1-z}\right)$ is convex for $z \in(0,1)$, since its first derivative,

$$
\frac{d}{d z} \ln \left(\frac{1}{1-z}\right)=-\frac{d}{d z} \ln (1-z)=\frac{1}{1-z}
$$

is increasing in $z$. Since in addition $\left.\ln \left(\frac{1}{1-z}\right)\right|_{z=0}=0$, we conclude that $\ln \left(\frac{1}{1-z}\right) / z$ is increasing in $z$. Since $z=\frac{w}{w+x}$ is increasing in $w$ and decreasing in $x$, the result follows. 